\title{
VALORACIÓN DE INDEPENDENCIA FUNCIONAL EN PACIENTES GERIÁTRICOS
}

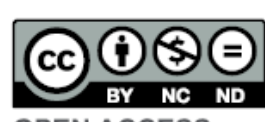

Este artículo está bajo una licencia de Creative Commons de tipo ReconocimienOPEN ACCESS to - No comercial - Sin obra

1. Pontificia Universidad Católica del Ecuador. Facultad de Enfermería. Carrera de Fisioterapia. Quito - Ecuador.

2. Universidad Internacional de la Rioja. Máster en Dirección y Gestión Sanitaria. La Rioja - España

ORCID ID:

Campoverde Francisco

orcid.org/0000-0003-0123-7758

Maldonado Jorge

orcid.org/0000-0002-0508-7286

*Corresponding author: Maldonado Jorge E-mail: jgmaldonado@uce.edu.ec

Article history

Received: 10 - Jul - 2020

Accepted: 02 - Sep - 2020

Publish: 01 - Oct - 2020

STROBE 2008 Check List statement: The

authors have real the STROBE 2008 Check List

and the manuscript was prepared and revised

according to the STROBE 2008 Checklist.

Conflict of interest: All authors declared that there are no conflicts of interest.

Financial disclosure: The authors have no financial relationships relevant to this article to disclose

Authors' contribution: All the authors analyzed and interpreted the data, drafted the article and critically revised the article. All the authors reviewed and approved the final manuscript.

\section{Resumen}

\section{Introducción}

Habitualmente las personas adultas mayores presentan cambios físicos, psicológicos, y sociales que afectan las funciones básicas debido al envejecimiento fisiológico. El objetivo de este estudio fue valorar el grado de independencia funcional en pacientes geriátricos y su relación con la edad, nivel de educación y tiempo de residencia.

\section{Pacientes y Métodos}

Se realizó un estudio descriptivo, transversal enfocándose en la valoración del grado de independencia funcional bajo el índice de Barthel; efectuado en residentes con rangos de edad entre 6590 años, donde se obtuvo una descripción importante del nivel de independencia en personas de la tercera edad. El total de la población (muestra) fue de 110 residentes (57 mujeres y 53 hombres).

\section{Resultados}

Pacientes del sexo masculino tuvieron mayor independencia funcional (43\% hombres vs. $32 \%$ mujeres), corroborando una apreciación estadística de un censo nacional hecho en el año 2010.

Adicionalmente, los resultados han sido analizados con sus correlaciones y las significancias del nivel de funcionalidad de los residentes respecto a su edad, nivel de educación, y tiempo de residencia.

\section{Conclusiones}

El nivel de instrucción mostró una ligera correlación con su nivel de funcionalidad, sin embargo, no se encontró relación entre la funcionalidad con la edad y/o tiempo de permanencia en el hogar. Futuros estudios a mayor escala deberían ser efectuados para un apoyo estadístico de relevancia.

Palabras clave: Valoración del Grado de Independencia Funcional, Índice de Barthel, Edad, Nivel de Educación, Tiempo de Residencia.
Forma de citar este artículo: Campoverde F, Maldonado J. VALORACION DE INDEPENDENCIA FUNCIONAL EN PACIENTES GERIÁTRICOS. Rev Med Vozandes. 2020; 31 (2): 56 - 64 


\begin{abstract}
Keywords: Functional Measure Independence, Barthel Index, age, education level, residence period.

\section{FUNCTIONAL INDEPENDENCE ASSESSMENT IN GERIATRICS PATIENTS}

\author{
Introduction \\ Older adults usually have physical, psychological, and social changes that affect basic \\ functions due to physiological aging. The objective of this study was to assess the degree \\ of functional independence in geriatric patients and their relationship to age, level of \\ education and time of residence.
}

Patients and Methods

A descriptive, cross-cutting study focused on the assessment of the degree of functional independence under barthel's index was carried out; made in residents with age ranges between 65-90 years, where an important description of the level of independence in seniors was obtained. The total population (sample) was 110 residents ( 57 women and 53 men).

Results

It showed that men have greater independence functionality (43\% men vs. $32 \%$ women), corroborating this appreciation with a national census statistic performed in 2010.

In addition, the results have been analyzed with its correlation and significance of the Residents'

Functional Level outcome regarding to their age, education level, and time in the institution.

\section{Conclusions}

The educational level showed a slight correlation with its physical functionality, however, no connection was found between functionality and age, nor institutionalized time. Further studies should be performed on a major scale to support static relevance.
\end{abstract}

\title{
Introducción
}

El concepto de envejecimiento puede variar dependiendo del autor, sin embargo, para dar a conocer un concepto científico y colectivamente adecuado, se lo definiría como un suceso presente a lo largo del ciclo vital desde el proceso de la concepción hasta la muerte. (1) Se lo describiría también, como el cambio fisiológico, psíquico, y social progresivo de una persona a medida que avanza en edad, lo que puede o no, llevar a cabo una repercusión del individuo para adaptarse física y psicológicamente con su entorno.

La razón que diferencia la calidad del envejecimiento poblacional saludable tendrá diversas causas, principalmente patológicas y de cuidado físico. Para el 2050 se prevé que mundialmente la población adulta mayor llegue a duplicarse. (2)

La OMS (Organización Mundial de la Salud) (3) refiere que llegar a una edad adulta mayor (60 años en adelante) con características físicas y cognitivas declaradas saludables, son producto de un aumento de la calidad de vida poblacional. independencia funcional posible y/o retrasar el grado de dependencia de un adulto mayor.

Los deterioros físicos generarán enfermedades de relevancia como son problemas cardiovasculares, sarcopenia, osteoporosis, artrosis, artritis, hipertensión los cuales no necesariamente están relacionados con el deterioro cognitivo, siendo propensos a tener dificultades para poder realizar actividades de la vida diaria y ser más dependientes después de los padecimientos antes mencionados.

Existe una correlación de la edad con la funcionalidad de la persona, ya que es un determinante de las pérdidas en las capacidades físicas. Hasta la edad de los 84 años la probabilidad de que una persona necesite ayuda se duplica, y entre los 85 y 94 años la necesidad se triplica. ${ }^{(4)}$

Los objetivos de los centros geriátricos son mantener la mayor La discapacidad es un término general que 
comprende deficiencias, limitaciones de actividades y restricciones de la participación. Las deficiencias son problemas que afectan a una estructura o función corporal; las dificultades para ejecutar acciones o tareas son las limitaciones de la actividad, y las restricciones de la participación son problemas para actuar en situaciones vitales. ${ }^{(5)}$

Esto conlleva a una dificultad en la ejecución de tareas básicas y produce problemas que intervienen en la participación significativa en medio de su ambiente social. Es una definición que refleja la interacción entre las características del organismo humano y la sociedad en la que vive. ${ }^{(5)}$

Las personas de la tercera edad son parte de poblaciones en riesgo o vulnerables en el Ecuador, por lo cual amerita proporcionar una atención prioritaria a este grupo. En la actualidad este conjunto de personas representa aproximadamente entre el 9 y 10 por ciento de la población total del país, y según datos estadísticos del INEC esta cifra aumentará progresivamente al tener una mayor esperanza de vida, lo que significaría un aumento relevante de adultos mayores en el futuro.

El objetivo de esta investigación fue valorar el grado de independencia funcional en los Residentes Adultos Mayores en el Hogar Corazón de María y además se describió los índices de clasificación de independencia funcional, respecto a su edad, sexo, y nivel de educación, siendo testeado mediante el Índice de Barthel.

Este trabajo expone en primera mano los cambios fisiológicos y repercusiones en la población geriátrica debido al proceso de envejecimiento fisiológico, posteriormente se procedió a describir los resultados de la valoración funcional aplicados por el Índice de Barthel y analizando las variables indicadas.

\section{Pacientes y Métodos}

\section{Selección de la cohorte}

Se realizó un estudio descriptivo, transversal, el cual fue llevado a cabo en el Hogar de Ancianos Corazón de María, ubicado en el norte de la ciudad de Quito y que brinda atención aproximadamente a 200 residentes que reciben atención diaria para cumplir sus necesidades básicas, servicio médico general y rehabilitación.

Se efectuó una entrevista directa a las personas implicadas en el análisis del presente trabajo, y por medio de datos básicos de filiación que fueron obtenidos de formularios recolectados por los investigadores para conseguir los antecedentes necesarios. Para ratificar la información que se obtuvo se utilizaron datos derivados de historias clínicas médicas, psicológicas, de trabajo social, mientras que para la medición del test aplicado se observaron revisiones bibliográficas, libros, artículos o artículos científicos, informes investigativos, reportes, y gray literatura que se involucraron en la materia.

Con relación al Índice de Barthel se procedió a realizar una lista de hombres y mujeres que entraron en el estudio, posteriormente se llevó a cabo la aplicación de la escala funcional con hojas de registro para anotar las puntuaciones de cada ítem de valoración, y para finalizar se efectuó la interpretación y análisis respectivo de los datos antes citados

\section{Métodos de evaluación funcional}

La técnica aplicada fue la observación directa y entrevista personal a cada adulto mayor incluido en el estudio y a sus cuidadores (auxiliares en enfermería). El instrumento que se utilizó para evaluar la dependencia 0 independencia funcional para las actividades de la vida diaria fue el uso del test Índice de Barthel.

Este test incluye los siguientes 10 ítems: comer, bañarse/lavarse, vestirse, arreglarse, deposición, micción, ir al retrete, trasladarse al sillón/cama, deambulación, subir y bajar escaleras. Todos estos ítems son valorados de diferente forma con puntuaciones entre $0,5,10$, y 15 puntos. ${ }^{(6)}$

Siendo interpretada de la siguiente forma:

- 0-20 dependiente total.

- 21-60 dependencia severa.

- 61-90 dependencia moderada.

- 91-99 dependencia escasa.

- 100 independencia.

El período de recolección de la información se realizó en los meses de agosto a septiembre del año 2014. Se trató de una muestra por conveniencia, pacientes adultos mayores de sexo masculino y femenino, con rangos de 65 a 90 años, con un estado de consciencia normal (lúcidos) y dispuestos a colaborar con el estudio fueron excluidos.

Adultos mayores que presentaban patologías neurológicas, demencia senil, ceguera, una discapacidad evidente, personas que presentaban alguna deformidadenextremidades superiores o inferiores (amputaciones) y aquellos que se encontraban en el área de Betania (psiquiatría) fueron excluidos.

El índice de Barthel se ha utilizado desde que fue propuesto en 1955 y ha dado lugar a múltiples versiones. Además de servir como estándar para la comparación con otras escalas. Las dos versiones principales del índice de Barthel, la de 10 ítems y la de 15 ítems, recibieron calificaciones altas de confiabilidad. Granger (1979) calculó una fiabilidad test - retest de 0,89 y una fiabilidad interna de 0,95 para la versión de 15 ítems, con cifras más altas obtenidas por Shinar (1987). El elemento de 10 tiene un coeficiente de consistencia interna alfa de 0,87 a 0,92 informado por Shah. En correlación con un índice de capacidad motora, el índice de Barthel recibió una calificación de 0.73 a 0.77 . Se acordó que el índice de Barthel es un instrumento confiable para prever la mortalidad y fácil de usar. (6) 


\section{Análisis estadístico}

Los datos obtenidos de la presente investigación fueron ingresados en una base electrónica en MS Excel y los resultados fueron expuestos en tablas y gráficos.

Para el análisis descriptivo, las variables cualitativas en esta investigación se expresaron en frecuencias y porcentajes, mientras que las variables cuantitativas, se manifestaron como promedios y desvíos estándar.

A su vez para el análisis inferencial de las variables cualitativas se utilizó la prueba de Chi cuadrado.

El valor de " $p$ " fue considerado como estadísticamente significativo si es igual o menor a 0,05.

Como instrumento estadístico el coeficiente de correlación de Pearson evaluó el grado de covarianza entre la variable de interés y las distintas variables explicativas. En ese sentido, se puede determinar en qué dirección (signo del coeficiente) y con qué fuerza (rango de -1 a 1) se da la relación lineal entre las variables de estudio.

\section{Aspectos éticos}

El presente estudio se manejó bajo las normas éticas básicas de la Declaración de Helsinki del año 2013, por lo que la información obtenida de los pacientes se trató exclusivamente para el desarrollo de la presente investigación y en el marco de la Historia Clínica manteniendo el carácter confidencial. La identidad de los pacientes es de absoluta reserva del investigador, sin la posibilidad de ser difundida a terceros, el contenido de los formularios por ningún motivo.

La autorización para la ejecución del estudio fue otorgada por parte de la dirección del Hogar de Ancianos Corazón de María (HACM), además se elaboró un consentimiento informado para la participación de los residentes. Este estudio sigue las directrices STROBE para estudios observacionales. ${ }^{(7)}$

\section{Resultados}

El estudio analizó las siguientes variables: distribución de edad, sexo, nivel de educación y tiempo de residencia en la Institución. De acuerdo con los criterios de inclusión y exclusión establecidos, la población de estudio fue de 110 personas, de las cuales fueron 57 mujeres (52\%) y 53 hombres (48\%), mismas que corresponden al $65 \%$ del total de la población estudio. Es importante observar que la categoría de edad de 75 a 84 años resultó ser el mayor rango porcentual mostrando $60 \%$ en hombres y $54 \%$ en mujeres como se aprecia en la Tabla1.

La distribución por nivel de educación se presenta en la tabla 1. Es interesante resaltar, que no se encontraron diferencias mayores por género, siendo ligeramente mayor el porcentaje de personas sin instrucción ( $40 \%$ en mujeres vs $38 \%$ en hombres), equivalente con primaria ( $47 \%$ en ambos), menos varones con instrucción secundaria ( $9 \%$ en hombres vs $12 \%$ en mujeres) e instrucción superior solamente en varones $16 \%$ en hombres vs. $0 \%$ en mujeres).
Con relación al tiempo de residencia en el Hogar, en la tabla 1 se presenta la distribución tanto en varones como en mujeres, en la cual se puede observarque el porcentaje que han permanecido más de 10 años es ligeramente mayor $(23 \%$ en mujeres vs $15 \%$ en hombres respectivamente; $p$ $=0.34$ ). Mientras que las categorías de rango de edad más bajas los porcentajes comparativos entre género son muy similares.

Tabla 1. Características clínicas y demográficas de los pacientes que participaron en el estudio categorizados según género.

\begin{tabular}{|lcl|}
\hline Variables & $\begin{array}{l}\text { Genero } \\
\text { Masculino, } \\
\text { n. (\%) }\end{array}$ & $\begin{array}{l}\text { Femenino, } \\
\text { n. (\%) }\end{array}$ \\
$65-74$ & $14(26 \%)$ & $8(14 \%)$ \\
$75-84$ & $32(60 \%)$ & $31(54 \%)$ \\
$85-90$ & $7(13 \%)$ & $18(32 \%)$ \\
TOTAL & $53(100 \%)$ & $57(100 \%)$ \\
Nivel de educación & & \\
Ninguna & $20(38 \%)$ & $23(40 \%)$ \\
Primaria & $25(47 \%)$ & $27(47 \%)$ \\
Secundaria & $5(9 \%)$ & $7(12 \%)$ \\
Superior & $3(6 \%)$ & $0(0 \%)$ \\
TOTAL & $53(100 \%)$ & $57(100 \%)$ \\
Tiempo Residencia (años) & \\
0-4 & $23(43 \%)$ & $24(42 \%)$ \\
5-9 & $22(42 \%)$ & $20(35 \%)$ \\
$\geq 10$ & $8(15 \%)$ & $13(23 \%)$ \\
TOTAL & $53(100 \%)$ & $57(100 \%)$ \\
\hline
\end{tabular}

\section{Métodos de evaluación funcional}

Se puede observar que en la valoración realizada mediante el índice de Barthel en los hombres existe un $43 \%$ que tiene independencia, a su vez el $2 \%$ tiene discapacidad severa. (Grafico 1)

Comparativamente se puede mencionar que las mujeres según la evaluación realizada el $51 \%$ tienen discapacidad moderada mostrando un valor más alto, mientras que el $3 \%$ tiene discapacidad severa revelando el valor más bajo. (Grafico 2)

En los gráficos $\mathbf{1}$ y $\mathbf{2}$ se evidencia que los hombres presentaron un mayor porcentaje de independencia funcional con respecto a las mujeres ( $43 \%$ vs $32 \%)$.

Los coeficientes reflejados en el (Gráfico 3) muestran una ligera tendencia positiva al relacionar el Nivel de Educación en hombres con el test. 


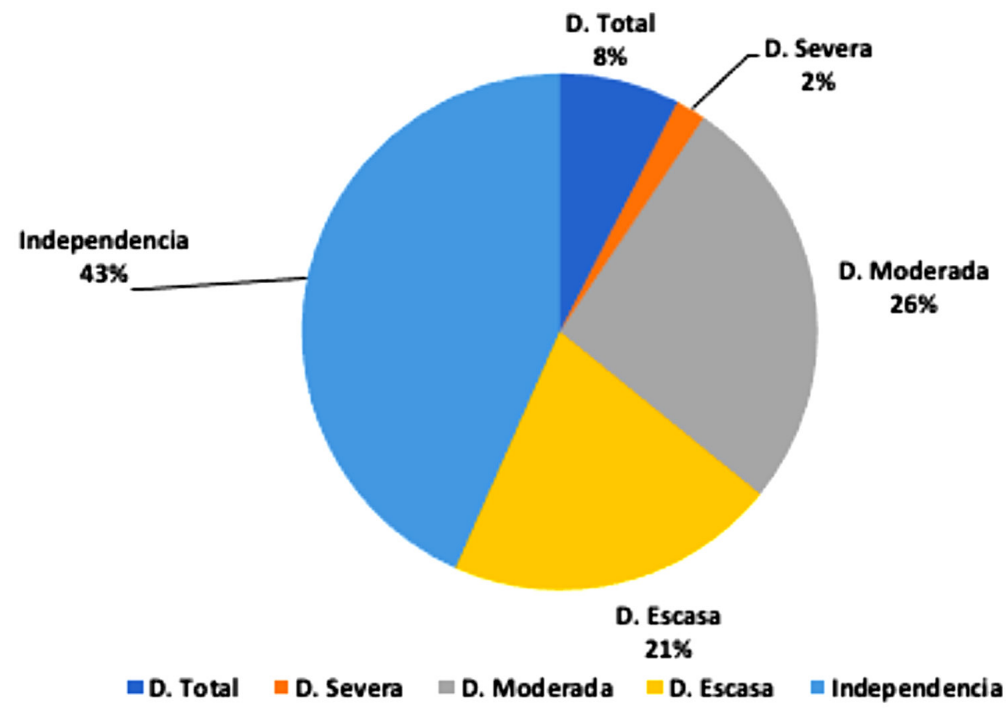

Gráfico 1. Índice de Barthel en Hombres

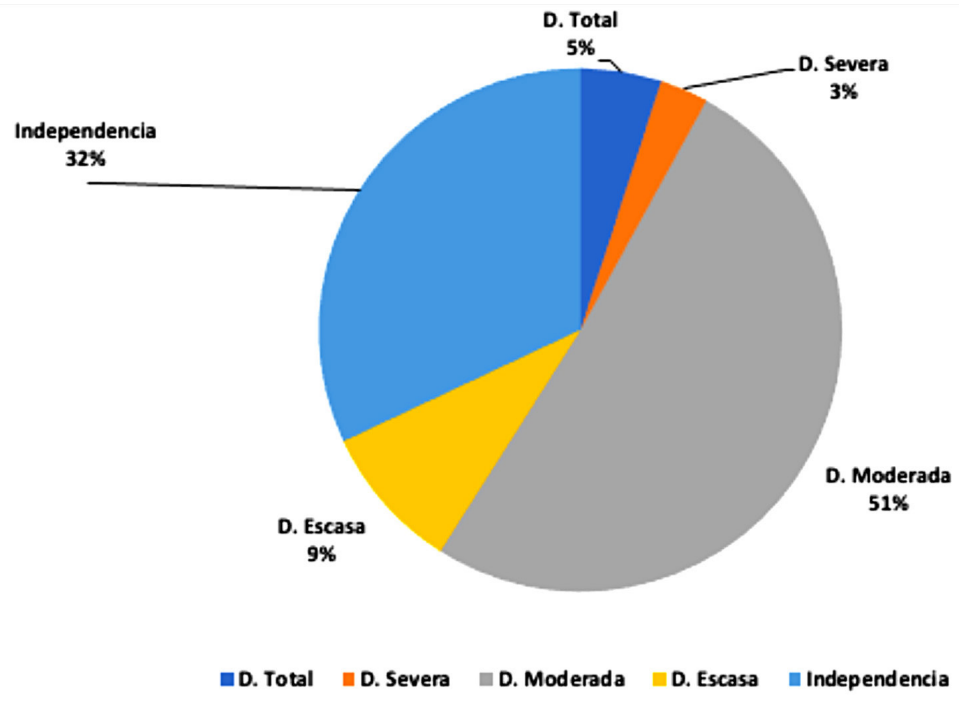

Gráfico 2. ndice de Barthel en Mujeres

Analizando la correlación entre el índice de Barthel y el nivel de educación en mujeres esta tendencia no tiene representatividad. (Gráfico 4).

Como se observa en la tabla 4 el total de independientes (4) personas), el $56 \%$ son hombres y el restante son mujeres.

Del total poblacional dependiente (69 personas), el mayor número son 39 mujeres

Además de las 110 personas del estudio, el grupo con mayor participación pertenece a las personas dependientes.

Del total poblacional (110), 88 personas se encuentran en el intervalo de edad de 75 años en delante siendo el grupo mayoritario, este dato es relevante para poder diferenciar el análisis estadístico.

Se puede observar que el primer grupo etario (74 años o menores) presenta un mayor número de personas independientes, sin embargo, en el segundo grupo etario se puede apreciar que el porcentaje dependiente es más elevado.

En la estadística inferencial de correlaciones como se observa en la Tabla 6, se comprobó que no existe correlación directa entre el Índice de Barthel y la Edad (hombres y mujeres) en el presente trabajo. 
Correlación Entre IB y Nivel de Educación (Hombres)

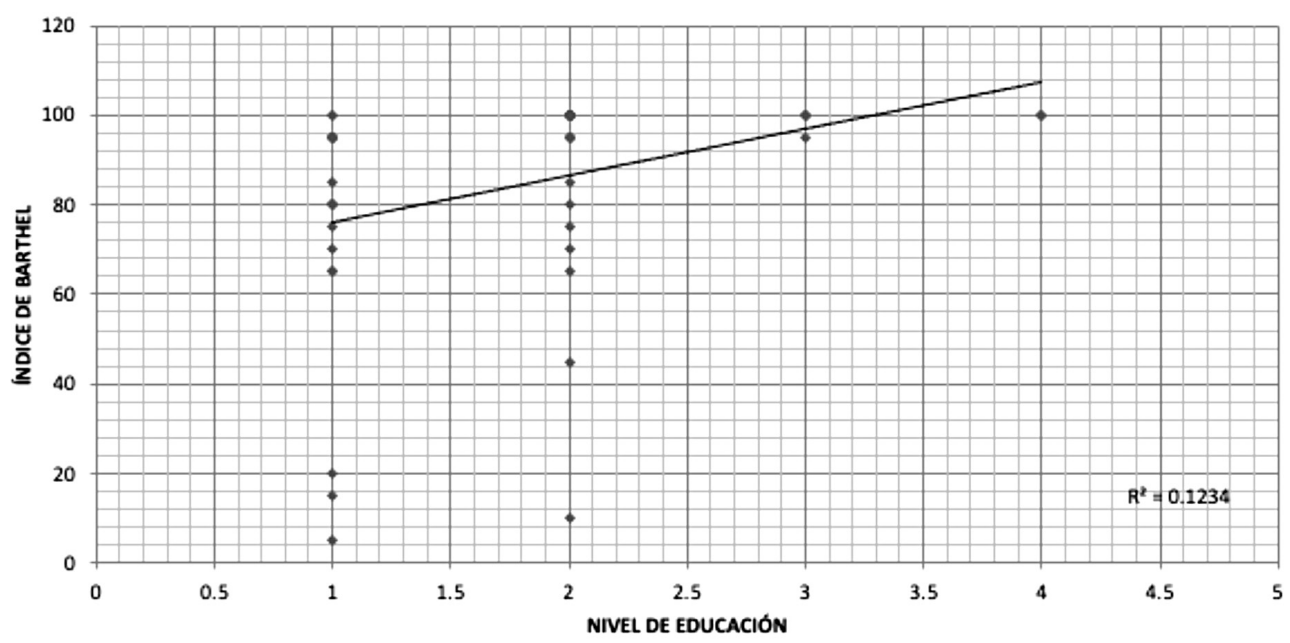

Gráfico 3. Correlación Entre Índice de Barthel y Fuente: Elaborado por los autores Nivel de Educación en Hombres

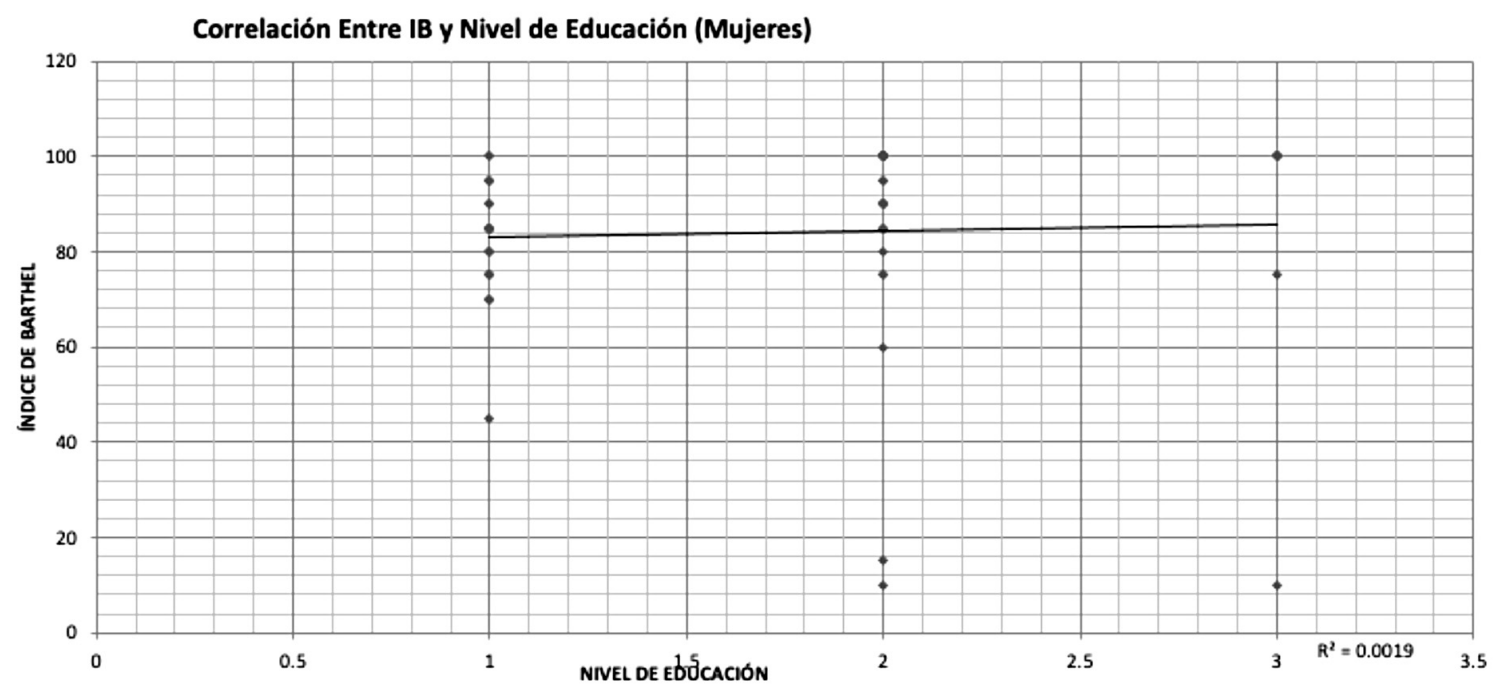

Gráfico 4. Correlación Entre Índice de Barthel y Fuente: Elaborado por los autores Nivel de Educación en Mujeres.

Tabla 2. Distribución entre Género y Funcionalidad

\begin{tabular}{|c|c|c|c|c|c|c|}
\hline \multirow[b]{2}{*}{ Hombres } & \multicolumn{2}{|c|}{ Independientes } & \multicolumn{3}{|c|}{ Dependientes } & \multirow{2}{*}{$\begin{array}{l}\text { Total } \\
\text { Génerc } \\
53\end{array}$} \\
\hline & $23\left(56^{*}\right)$ & {$\left[43^{* *}\right]$} & 30 & $(43 *)$ & {$\left[57^{* *}\right]$} & \\
\hline Mujeres & $18\left(44^{*}\right)$ & {$\left[32^{* *}\right]$} & 39 & $\left(57^{*}\right)$ & {$\left[68^{* *}\right]$} & 57 \\
\hline $\begin{array}{l}\text { Total Funcio- } \\
\text { nalidad }\end{array}$ & 41 & & 69 & & & 110 \\
\hline
\end{tabular}

*Los valores representados en paréntesis pertenece a los porcentajes calculados para la categoría de funcionalidad

**Los valores pertenecen a los porcentajes de la categoría de Género Fuente: Elaborado por los autores

Al realizar el mismo análisis, pero usando el Nivel de Educación (hombres y mujeres), se encontró una ligera tendencia de relación en el caso de los varones, pero dicha correlación estuvo ausente en el grupo de las mujeres.
Y por último no se pudo establecer correlación entre el índice de Barthel y el tiempo de residencia en el hogar.

Como se puede observar en la tabla 5 el valor de chi cuadrado es de 1.64 en Género y Funcionalidad. El valor de $P$ es igual a 0.200273. Este resultado no es significativo, por lo que la diferencia en el Nivel de Independencia Funcional por el Índice de Barthel entre Hombres y Mujeres no es extrapolable.

Según la estadística inferencial Chi cuadrado de la Tabla 5, se presentan en la primera variable de Género y Funcionalidad un resultado que no es significativo, por lo que la diferencia en el Nivel de Independencia Funcional por el Índice de Barthel entre Hombres y Mujeres no es reproducible. 
Tabla 3. Distribución entre Grupo Ełario y Funcionalidad

\begin{tabular}{|c|c|c|c|}
\hline & Independientes & Dependientes & $\begin{array}{l}\text { Total } \\
\text { Edad }\end{array}$ \\
\hline $\begin{array}{l}74 \text { años o } \\
\text { menores }\end{array}$ & $12 \quad(29 *) \quad\left[55^{* *}\right]$ & $10 \quad\left(15^{*}\right) \quad\left[45^{* *}\right]$ & 22 \\
\hline $\begin{array}{l}75 \text { años en } \\
\text { adelante }\end{array}$ & $29 \quad(71 *) \quad\left[33^{* *}\right]$ & $59 \quad\left(85^{*}\right) \quad\left[67^{* *}\right]$ & 88 \\
\hline $\begin{array}{l}\text { Total Funcio- } \\
\text { nalidad }\end{array}$ & 41 & 69 & 110 \\
\hline
\end{tabular}

*Los valores representados en paréntesis pertenece a los porcentajes calculados para la categoría de funcionalidad **Los valores pertenecen a los porcentajes de la categoría de Género Fuente: Elaborado por los autores

En la segunda variable el resultado de edad en hombres, mujeres y funcionalidad es casi significativo, lo que sugiere que la diferencia en el Índice de Barthel podría guardar relación con las edades, pero por el enfoque del estudio y tamaño de la muestra no es posible observarlo.

\section{Tabla 4. Distribución entre Grupo Etario y Funcionalidad}

\begin{tabular}{|ll|}
\hline VARIABLE & $\mathbf{R}^{2}$ \\
IB y Edad Hombres & $\mathrm{R}^{2}=0.0036$ \\
IB y Nivel Educación Hombres & $\mathrm{R}^{2}=0.1234$ \\
IB y Tiempo de Residencia (H y M) & $\mathrm{R}^{2}=0.0008$ \\
IB y Edad Mujeres & $\mathrm{R}^{2}=0.0054$ \\
IB y Nivel Educación Mujeres & $\mathrm{R}^{2}=0.0019$ \\
Fuente: Elaborado por los autores & \\
\hline
\end{tabular}

Fuente: Elaborado por los autores

Tabla 5. Estadística Inferencial - Chi cuadrado

\begin{tabular}{|c|c|c|c|}
\hline $\mathbf{N}^{\circ}$ & VARIABLE & $\begin{array}{l}\text { CHI } \\
\text { CUADRADO }\end{array}$ & VALOR P \\
\hline 1 & Género y Funcionalidad & 1.64 & 0.0200273 \\
\hline 2 & $\begin{array}{l}\text { Edad (H y M) y } \\
\text { Funcionalidad }\end{array}$ & 3.5092 & 0.061029 \\
\hline 3 & Edad (M) y Funcionalidad & 8.1205 & 0.004377 \\
\hline 4 & Edad (H) y Funcionalidad & 0.0023 & 0.96216 \\
\hline 5 & $\begin{array}{l}\text { Tiempo de Residencia } \\
(\mathrm{H} \text { y } \mathrm{M})\end{array}$ & 3.1916 & 0.074015 \\
\hline
\end{tabular}

Fuente: Elaborado por los autores

El valor de chi cuadrado de la edad hombres y mujeres con su funcionalidad es 3.5092 y el valor de P es igual a 0.061029 como se puede apreciar en la tabla 5. Este resultado es casi significativo, lo que sugiere que la diferencia en el Índice de Barthel podría guardar relación con las edades, pero por el tamaño de la muestra no es posible manifestarlo.

En la tercera variable edad de mujeres y funcionalidad, si existe una relación significativa en las mujeres comparadas con la funcionalidad por el Índice de Barthel y la edad, pero en la cuarta variable la edad de hombres y funcionalidad en el
Índice de Barthel no es significativa y por lo tanto solo aplica para esta investigación.

En la quinta y última variable el resultado muestra que la diferencia encontrada en el Índice de Barthel con relación al tiempo de residencia de los Adultos Mayores no es significativa y por lo tanto también solo es aplicable para este estudio.

\section{Tabla 6. Estadística inferencial - Correlaciones}

\begin{tabular}{|ll|}
\hline VARIABLE & $\mathbf{R}^{2}$ \\
IB y Edad Hombres & $\mathrm{R}^{2}=0.0036$ \\
IB y Nivel Educación Hombres & $\mathrm{R}^{2}=0.1234$ \\
IB y Tiempo de Residencia (H y M) & $\mathrm{R}^{2}=0.0008$ \\
IB y Edad Mujeres & $\mathrm{R}^{2}=0.0054$ \\
IB y Nivel Educación Mujeres & $\mathrm{R}^{2}=0.0019$ \\
\hline
\end{tabular}

Fuente: Elaborado por los autores

Tabla 7. Estadística Inferencial - Chi cuadrado

\begin{tabular}{|llll|}
\hline $\mathbf{N}^{\circ}$ & VARIABLE & $\begin{array}{l}\text { CHI } \\
\text { CUADRADO }\end{array}$ & VALOR P \\
$\mathbf{1}$ & $\begin{array}{l}\text { Género y } \\
\text { Funcionalidad }\end{array}$ & 1.64 & 0.0200273 \\
$\mathbf{2}$ & $\begin{array}{l}\text { Edad (H y M) y } \\
\text { Funcionalidad }\end{array}$ & 3.5092 & 0.061029 \\
$\mathbf{3}$ & $\begin{array}{l}\text { Edad (M) y } \\
\text { Funcionalidad }\end{array}$ & 8.1205 & 0.004377 \\
$\mathbf{4}$ & $\begin{array}{l}\text { Edad (H) y } \\
\text { Funcionalidad }\end{array}$ & 0.0023 & 0.96216 \\
$\mathbf{5}$ & $\begin{array}{l}\text { Tiempo de Resi- } \\
\text { dencia (H y M) }\end{array}$ & 3.1916 & 0.074015 \\
\hline
\end{tabular}

Fuente: Elaborado por los autores

\section{Discusión}

En los resultados los hombres presentan un mayor porcentaje de independencia funcional que las mujeres ( $43 \%$ vs $32 \%$ respectivamente). Esto se corrobra en un estudio donde las personas adultas mayores en el Ecuador muestran que la población femenina evidencia un mayor nivel de afectación de discapacidad y menor función que los hombres. El $21 \%$ de las mujeres y el $43 \%$ de los hombres ingresan en un grupo perfectamente funcional, mientras que el $19 \%$ de las mujeres y el $13 \%$ de los hombres sufren de discapacidad. ${ }^{(8)}$

Freitas R, Coqueiro R, Junior W(9) atribuyen que, significativamente, existe un mayor nivel de dependencia en las Actividades Instrumentales de la Vida Diaria en adultos seniles de sexo femenino mayores a 80 años que no tenían ningún nivel de escolaridad (analfabetismo). En otros estudios se presentan los siguientes hallazgos a ser contrastados, en uno manifestaban que un menor nivel de educación era asociado con una 
baja actividad laboral y física, lo que provoca una repercusión física en adultos mayores. ${ }^{(10)}$ De igual forma, otra investigación se expone que mujeres adultas mayores con mayor educación obtuvieron una mayor puntuación en una prueba de funcionalidad "lamada "timed get up and go" test. (11)

En el aspecto educativo, se puede sugerir que a mayor instrucción tendrá una mayor funcionalidad, debido a que va aumentando progresivamente el nivel de educación y a su vez aumentará el puntaje que tiene la persona en el Índice de Barthel, esto podría deberse cuando la formación de un individuo es alta tiende a mostrar mayor cultura, por lo tanto, la persona estará más activa en realizar tareas de interés u ocio. Pero hay que tomar en cuenta que esta aseveración no es general, ya que existen residentes que no pudieron tener educación alguna, sin embargo, tienen un nivel funcional moderado o alto, lo que da a entender que los hábitos de la persona son los que determinan sus capacidades funcionales, pero su nivel educativo puede influir de cierta manera.

Al pretender identificar si la edad de los hombres o las mujeres guarda relación con el índice de Barthel, se demostró que en ninguno de los casos existe una correlación directa ni significativa en este trabajo, sin embargo, varios estudios demuestran que la edad influye notablemente en la disminución de la actividad y estado físico de la persona. ${ }^{(12)}$

En la estadística inferencial se mostró que la significancia fue mínima en las tablas de las correlaciones, mientras que los valores de chi cuadrado no mostraron una relación representativa; surgió una concordancia entre funcionalidad, edad en hombres y mujeres en conjunto, pero al ser analizados por separado, existe una relación significativa con las mujeres, lo cual podría inferir que la independencia funcional es independiente del sexo.

Colón C, Pavon J (13) mencionan que a mayor edad existe una menor funcionalidad en adultos mayores, sin embargo, estos efectos en el cuerpo serían menores si se realiza un plan de tratamiento preventivo dirigido a la administración de fármacos y protocolos de fisioterapia adecuados. Además, Zurita, et al.. (14) coincide con este planteamiento, en donde se manifestó que existe una relación proporcional, en cuánto a mayor edad, menor puntuación de funcionalidad tendrá un adulto mayor.

Finalmente no se estableció correlación entre el índice de Barthel y el tiempo de Residencia en el Hogar, pero Quadri, et al., (15) evidenciaron que el tiempo de residencia en un asilo es un predictor importante en la disminución de independencia funcional, se evaluaron varios niveles de funcionalidad mediante 3 diferentes tipos de test, demostrando una relación que al tener una menor puntuación hay menor probabilidad de mantener las funciones básicas de la vida diaria durante años posteriores de institucionalización.

Mientras que Guillén F, Pérez J, Petidier $\mathrm{R}^{(16)}$ indican que las personas adultas mayores que viven en residencias geriátricas sufren más incapacidades a largo plazo, mostrando que un $70 \%$ precisa ayuda en tareas para el servicio sanitario y traslados de la cama a una silla, 50\% presenta incontinencia intestinal o vesical, y $40 \%$ requiere ayuda para comer.
Respecto al tiempo de residencia y edad existe una relación con el Índice de Barthel, donde muestra que a mayor tiempo de residencia y edad existe menor valoración en la independencia funcional.

La derivación de este trabajo puede relacionar los cambios degenerativos físicos (posturales), sensoperceptivos (equilibrio), musculo esqueléticos y psicosociales que influyen de alguna o varias formas en la clasificación funcional que presenta un adulto mayor en el Hogar Ancianos Corazón de María. Por ejemplo, una persona tiene dificultades para ducharse por sí mismo por razones como pérdida de masa muscular, otras precisan adaptaciones ortopédicas como un asiento para bañarse sentados por la falta de equilibrio, en el aspecto psicológico presentan miedos al asearse solos por una recidiva a caídas. Así existen soluciones como asistencia al momento de ir al retrete, en donde algunas personas necesitan de ayuda para ir y regresar del inodoro como precaución para evitar una caída porque la persona tiene inestabilidad sensorial. Otros utilizan apoyos ortésicos para la deambulación, como bastones y andadores por enfermedades degenerativas óseas o por problemas posturales y ergonómicos.

La falta de participación en las actividades lúdicas de las personas provoca un aislamiento social y sentimiento de soledad. Esto a veces promueve que un residente senil sea propenso a presentar un estado anímico bajo para realizar actividad física, lo que acarrea un sedentarismo, que a corto plazo no puede ser relevante, pero si a largo plazo ocasionará inconvenientes muy simples que pueden ir aumentando e influyendo en las actividades de la vida diaria.

Según el análisis de este estudio podemos observar que para los próximos años la población de adultos mayores del país crecerá, por ende, es imprescindible la proyección de instituciones con mayor especialidad en el cuidado de la población geriátrica.

Estos grupos vulnerables, requieren atenciones más ecuánimes del personal de salud desde diversos procedimientos para ejecutar tratamientos preventivos y desarrollar políticas públicas que impulsen a su cuidado de acuerdo con las características físicas y emocionales que se presentan en este grupo etario.

Este trabajo debería ser una base para realizar un estudio a mayor escala y ver si existen significancias estadísticas que no se logró, para eso se puede trabajar con otras instituciones, centros y hospitales para promover a otras personas interesadas y relacionadas al estudio a efectuaruna investigación similar en donde se puedan comparar y refutar los 
datos de este trabajo para observar si los resultados pueden ser replicables.

Al final de este estudio se ven datos establecidos por el envejecimiento fisiológico del ser humano, sin embargo, es posible cambiar nuestro estilo de vida realizar ejercicio físico y mental, tener una alimentación saludable, y preservar las relaciones sociales, puede lograr beneficios importantes a largo plazo a medida que el individuo envejece.

\section{Referencias}

1. Ruíz D. Psicología del envejecimiento. Fundamentals of Geriatrics Medicine. Medellín: Corporación para Investigaciones Biológicas CIB; 2006, pp. 64-8.

2. Montenegro E, Cuellar F. Geriatrics and Gerontology for the Internist Physician. la ed. Grupo Editorial La Hoguera; 2012.

3. World Health Organization. 10 facts on ageing and health [Internet]. 2020. [citado el 15 de febrero de 2020]. Disponible en: http://www. who.int/features/factfiles/ageing/es/

4. Salgado A , Guillén F, Ruipérez I. Geriatrics Manual. $3^{a}$ ed. Masson; 2003.

5. World Health Organization.Disability and Health [Internet]. 2020 [citado el 18 de febrero de 2020]. Disponible en: http://www.who.int/ mediacentre/factsheets/fs352/es/

6. Florence I. Barthel Index (BI) Statistics Solutions. [Internet]. 2020 [citado el 18 de septiembre de 2020].Disponible en: https:// www.statisticssolutions.com/barthelindexbi/\#: : text=Reliability\%20 and $\% 20$ Validity\&text=Correlated\%20with\%20a\%20 Validity\&text=Correlated\%20with\%20a\%20
motor\% 20 ability, mortality\% 20 and $\% 20$ easy\%20to\%20use
7. von Elm E, Altman DG, Egger M, Pocock SJ, Gotzsche PC, Vandenbroucke JP; STROBE Initiative. The Strengthening the Reporting of Observational Studies in Epidemiology (STROBE) statement: guidelines for reporting observational studies. J Clin Epidemiol. ting observational studies. J Clin Epidemiol.
2008 Apr;61 (4):344-9. doi: 10.1016/j.jcline2008 Apr;61 (4):3
pi.2007.11.008

8. Ministry of Economic and Social Inclusion. National Survey of Health, Well-being and Aging. Quito: Grupo Impresor: 2010.

9. Freitas R, Coqueiro R, Junior W. Functional capacity and associated factors in the elderly: a population study. Scielo. 2012;.25 (6)

10. Shaw B, Spokane L. Examining the Association Between Education Level and

Physical Activity Changes During Early Old Age. 2008 [citado el 20 de marzo de 2020]. Disponible en: http://journals.sagepub.com/doi/ nible en: http://journals.sagepub.com/doi/
abs/10.1177/0898264308321081 ?url ver=Z39.8 abs/10.1177/0898264308321081?url_ver $=Z 39.8$
- $2003 \&$ rfr_id=ori:rid:crossref.org\&rfr_dat $=C_{-}$ pub\%3dpubmed

11. Gomes C, Teixeira L, Fonseca, Freitas S. Obtenido de Idade e educação influenciam o desempenho de mulheres idosas no teste Timed
Up and Go com dupla tarefa. Scielo. 2015 [citado el 28 de marzo de 2020]. Disponible en: http://www.scielo.br/scielo.php? script=sci arttext\&pid=S0004-282X2015000300187

12. Milanovic Z, Pantelic S, Trajkovic N. Agerelated decrease in physical activity and functional fitness among elderly men and women. Clinical Interventions in Aging. 2013 p. 549-556

13. Colón E, Pavon, J. Functional Decline in OIder Adults. American Family Physician. 2013; $88(6): 388-394$

14. Zurita D. Daily activity according to Barthel in dex in older adults Ibarra. Nursing Investigate. 2015; 1 (3).

15. Quadri P, Tettamant M, Bernasconi S, Trento $F$, Loew F. Lower limb function as predictor of falls and loss of mobility with social repercussions one year after discharge among elderly inpatients. Aging Clin Exp Res. 2005; 17: 82 89.

16. Guillén F, Pérez J, Petidier R. Syndromes and Care in the Geriatric Patient. $2^{a}$ ed. Elsevier Masson; 2008. 